\author{
Department of Physics and Astronomy \\ Experimental Particle Physics Group \\ Kelvin Building, University of Glasgow, \\ Glasgow, G12 8QQ, Scotland \\ Telephone: +44 (0)1413302000 Fax: +44 (0)141330 5881
}

\title{
Prospects for CP Violation studies at $\mathrm{LHCb}$
}

\author{
Vladimir Vava Gligorov ${ }^{1}$ \\ On behalf of the LHCb collaboration \\ ${ }^{1}$ University of Glasgow, Glasgow, G12 8QQ, Scotland \\ E-mail: v.gligorov@physics.gla.ac.uk
}

\begin{abstract}
$\mathrm{LHCb}$ is the dedicated $B$ physics experiment at the Large Hadron Collider, whose goals are to overconstrain the CKM matrix and search for New Physics in the flavour sector. This contribution describes LHCb's programme for CP violation measurements. LHCb is introduced in the context of the LHC, and the current knowledge of CP violation is discussed. Three key areas of LHCb's physics programme are looked at in more detail: measurements of the CKM angle $\gamma$, measurements of the $B_{s}^{0}$ mixing phase $\phi_{s}$, and searches for New Physics in $b \rightarrow s \bar{s} s$ decays. LHCb's potential to measure the CKM angles $\alpha$ and $\beta$ is also discussed.
\end{abstract}

Lake Louise Winter Institute 2008

Lake Louise, Alberta, Canada 


\section{Introduction}

\subsection{The LHCb Experiment at the Large Hadron Collider}

The Large Hadron Collider [1] at CERN is a proton-proton collider which will operate at a centre-of-mass energy of $14 \mathrm{TeV}$, with a maximum luminosity of $10^{34} \mathrm{~cm}^{-2} \mathrm{~s}^{-1}$. LHCb [2] is a dedicated $B$ physics experiment which will exploit the unprecedented quantity of $b$ hadrons produced at the LHC to over-constrain the CKM matrix and search for New Physics (NP) in the flavour sector. It will take data at a luminosity of $2 \cdot 10^{32}$ $\mathrm{cm}^{-2} \mathrm{~s}^{-1}$.

The LHC's $b \bar{b}$ cross section of $\sim 500 \mu \mathrm{b}$ means that LHCb will have a statistical reach unmatched by any previous $B$ physics experiment, while the centre-of-mass energy of $14 \mathrm{TeV}$ gives it unique access to all flavours of $B$ mesons and baryons. In addition, the produced $B$ mesons are highly boosted. LHCb will achieve a typical lifetime resolution of $40 \mathrm{fs}$, allowing for precision measurements of time dependent $C P$ asymmetries in the neutral $B$ sector.

The current status of both the LHC collider and the LHCb experiment are described in more detail by other contributions in these proceedings.

\subsection{Current knowledge of CP violation}

Although knowledge of the CKM matrix, which describes CP violation in the Standard Model (SM), has improved significantly in recent years, several of its parameters remain poorly constrained by direct measurements. Chief among these are the CKM angle $\gamma$ and the $B_{s}^{0}$ mixing phase $\phi_{s}$. The CKM Fitter[3] average of $\gamma$ from direct measurements is

$$
\gamma=76.8^{\circ}[+30.4-31.5]^{\circ}
$$

which can be compared to the global SM constraint

$$
\gamma=67.6[+2.8-4.5]^{\circ}
$$

While $\phi_{s}$ is predicted to be very small in the SM

$$
\phi_{s}=-0.0368 \pm 0.0018 \mathrm{rad},
$$

the tightest direct constraint on its value at the time of this conference came from the D0 measurement[4]

$$
\phi_{s}=-0.79 \pm 0.56_{-0.14}^{+0.01} \mathrm{rad}
$$

Making precision measurements of these parameters is a key goal of LHCb, as any deviation from their SM values would be a clear sign of NP.

\section{Measurements of the CKM angle $\gamma$}

\subsection{Measuring $\gamma$ from $B_{d, s}^{0} \rightarrow h h$ decays}

The $B^{0} \rightarrow h h$ family $^{1)}$ of decays have decay rates with non-negligible contributions from penguin diagrams, which make them sensitive to NP.

The dependence on $\gamma$ comes from the time-dependent $\mathrm{CP}$ asymmetries in the $B_{s}^{0} \rightarrow K K$ and $B_{d}^{0} \rightarrow \pi \pi$ decays. However, these asymmetries also depend on the amplitude ratio of the penguin and tree decay diagrams $d_{h h} e^{i \theta_{h h}}$, as well as the mixing phases $\phi_{d}$ and $\phi_{s}$. Since there are four asymmetries and seven unknown parameters, it is necessary to employ U-spin symmetry, which leads to $d_{\pi \pi} \equiv d_{K K}$ and $\theta_{\pi \pi} \equiv \theta_{K K}$. This allows $\gamma$ to be measured when combined with external constraints on $\phi_{d}$ and $\phi_{s}$.

LHCb will reconstruct[5] $36 k B_{s}^{0} \rightarrow K K$ and $36 k B_{d}^{0} \rightarrow \pi \pi$ decays with $2 f b^{-1}$ of data taking, with $B / S$ ratios of 0.06 and 0.46 respectively. These yields allow $\gamma$ to be determined ${ }^{2)}$ with a precision of $10^{\circ}$.

\subsection{Measuring $\gamma$ from $B^{ \pm} \rightarrow D^{0} K^{ \pm}$decays}

The charmed decays of charged $B$ mesons proceed through tree-level diagrams, and enable a direct SM measurement of $\gamma$. First measurements of this kind have already been made at the B factories[6], and are the inputs to the global average on $\gamma$ quoted above.

\footnotetext{
1) Where $B^{0}$ stands for $B_{d}^{0}$ or $B_{s}^{0}$ and $h$ stands for a $\pi$ or $K$ meson.

2) Allowing for a $20 \%$ level of U-spin breaking.
} 
Different strategies exist for measuring $\gamma$, depending on the final state into which the $D^{0}$ decays. In the GLW $[7,8]$ strategy, the $D^{0}$ decays into a CP eigenstate, and the sensitivity to $\gamma$ comes from the interference between dominant and doubly colour-suppressed decays. The ADS[9] strategy combines colour-suppressed B decays with colour-favoured D decays (and vice versa), thus increasing the interference effects. The GGSZ[10] strategy uses a Dalitz analysis of $D^{0} \rightarrow K_{S} \pi \pi$ decays to extract $\gamma$ together with the strong phases in the $D^{0}$ decay. It is expected[11, 12, 13, 14] that these methods, when combined, will measure $\gamma$ to $\sim 5^{\circ}$ with $2 f b^{-1}$ of data taking at LHCb.

\subsection{Measuring $\gamma$ from $B_{s, d}^{0} \rightarrow D_{s, d}^{ \pm}\left(K^{\mp}, \pi^{\mp}\right)$ decays}

The time dependent $\mathrm{CP}$ asymmetries in the tree level decays $B_{s}^{0} \rightarrow D_{s}^{ \pm} K^{\mp}$ and $B_{d}^{0} \rightarrow D^{ \pm} \pi^{\mp}$ can be used to measure the SM value of $\gamma+\phi_{s, d}$. The measured value will, in principle, suffer from an eightfold ambiguity. Together with the measurement of $\gamma$ from charged $B$ decays, they will provide a baseline SM measurement of $\gamma$; this will allow any NP effects in the measurement of $\gamma$ from $B^{0} \rightarrow h h$ decays to be constrained.

The expected sizable lifetime difference $\Delta \Gamma_{s}$ in the $B_{s}^{0}$ system allows for most of these ambiguities to be resolved when the flavour specific $B_{s}^{0} \rightarrow D_{s}^{-} \pi^{+}$decays are used to help constrain $\Delta \Gamma_{s}$ and $\Delta m_{s}$. A yield[15] of $6.2 k B_{s}^{0} \rightarrow D_{s}^{ \pm} K^{\mp}$ events is expected with $2 \mathrm{fb}^{-1}$ of data taking, with a $B / S$ of 0.7 , leading to a statistical precision of $10^{\circ}$ on $\gamma$.

In contrast with the $B_{s}^{0}$ mesons, no sizable lifetime difference is expected in the $B_{d}^{0}$ system and the ambiguities remain. In addition, the interference term in the $B_{d}^{0}$ decays is too small to fit directly from the CP asymmetries and will have to be externally constrained. Both of these problems can be solved by relating the decays $B_{s}^{0} \rightarrow D_{s}^{ \pm} K^{\mp}$ and $B_{d}^{0} \rightarrow D^{ \pm} \pi^{\mp}$ through U-spin symmetry[18]. A fully unambiguous extraction of $\gamma$, with a precision better than $10^{\circ}$, is expected $[16,17]$ with $2 f b^{-1}$ of data taking.

\section{Measuring the $B_{s}^{0}$ mixing phase $\phi_{s}$}

Although the $B_{s}^{0}$ mixing phase $\phi_{s}$ is very small in the SM, it can receive sizable NP contributions through box diagrams involving top quark exchange. The golden mode for measuring $\phi_{s}$ at $\mathrm{LHCb}$ is $B_{s}^{0} \rightarrow J / \psi \phi$, which is expected to have a yield[19] of $130 k$ events with $2 \mathrm{fb}^{-1}$ of data taking, with a $B / S$ of 0.12 .

The measurement of $\phi_{s}$ from the time dependent decay rate asymmetries is complicated by the fact that $B_{s}^{0} \rightarrow J / \psi \phi$ is not a pure CP mode. CP-even and CP-odd contributions can be separated by studying their distributions in the transversity angle, and a precision of $0.023 \mathrm{rad}$ can be achieved on $\phi_{s}$ with $2 \mathrm{fb}^{-1}$ of data taking. After $10 \mathrm{fb}^{-1}$ of data taking, LHCb will have $>3 \sigma$ evidence of a non-zero $\phi_{s}$ even if no new physics is discovered. In addition, other CP-specific final states will be measured[20], leading to a small improvement in the overall precision.

\section{CP violation in $b \rightarrow s \bar{s} s$ decays}

The $B_{s}^{0} \rightarrow \phi \phi$ and $B_{d}^{0} \rightarrow \phi K_{S}$ decays proceed through penguin diagrams, making them sensitive to NP. CP violation in these decays is $<1 \%$ in the SM because of cancellation between the mixing and penguin phases. These phases can be affected differently by NP, leading to a significant CP violation measurement at LHCb. The measurement of $\phi_{s}$ from $B_{s}^{0} \rightarrow J / \psi \phi$ discussed above will allow NP contributions from mixing and penguins to be disentangled.

The decay $B_{s}^{0} \rightarrow \phi \phi$ is expected to yield[21] $3.1 k$ events with $2 f b^{-1}$ of data taking, with a $B / S<0.8$ at $90 \% \mathrm{CL}$. A statistical precision of $0.1 \mathrm{rad}$ is expected on the total weak phase, and a measurement significantly different from zero would be a clear signal of NP. The decay $B_{d}^{0} \rightarrow \phi K_{S}$ is expected to yield[22] 920 signal events with $2 \mathrm{fb}^{-1}$ of data taking, with a $B / S<1$ at $90 \% \mathrm{CL}$. This affords a precision of $\sigma_{S}=0.23$ on the mixing-induced $\mathrm{CP}$ asymmetry and $\sigma_{C}=0.29$ on the direct CP-asymmetry.

\section{$5 \quad$ Other measurements: $\alpha, \sin (2 \beta)$}

LHCb will measure $\sin (2 \beta)$ from the channel $B_{d}^{0} \rightarrow J / \psi K_{S}$. The expected yield[23] is $236 k$ events with $2 f b^{-1}$ of data taking, leading to a precision of 0.020 on $\sin (2 \beta)$.

$\mathrm{LHCb}$ can make an unambiguous measurement of the angle $\alpha$ from the $B_{d}^{0} \rightarrow(\rho \pi)^{0}$ decays, which have an expected yield[24] of $14 k$ events with $2 f b^{-1}$ of data taking with a $B / S$ ratio of 1 . A precision of $8.5^{\circ}$ on $\alpha$ is expected with such a data sample, with a $15 \%$ chance for the fit to converge on a pseudo-mirror solution. This chance is reduced to less than $1 \%$ with $10 \mathrm{fb}^{-1}$ of data taking, and the statistical precision is reduced to $\sim 5^{\circ}$. 


\section{Conclusion}

As the dedicated flavour physics experiment at the LHC, LHCb has significant potential to discover NP. LHCb will make precision measurements of the CKM angle $\gamma$, the $B_{s}^{0}$ mixing phase $\phi_{s}$, and the effective mixing phase in $b \rightarrow s \bar{s} s$ decays. These measurements will complete the SM picture of CP violation and constrain any NP seen in the flavour sector.

\section{Acknowledgements}

The author wishes to acknowledge the helpful suggestions made by F. Mueheim and G. Wilkinson during the preparation of this contribution.

\section{References}

[1] LHC Study Group, CERN/AC/90-05(LHC) (1995).

[2] The LHCb Collaboration, The LHCb detector at LHC, to be published in JINST (2008).

[3] CKMfitter Group (J. Charles et al.), Eur. Phys. J. C41, 1-131 (2005).

[4] V. M. Abazov et. al., D0 Collaboration, Phys. Rev. Lett. 98, 121801 (2007)

[5] A. Carbone, J. Nardulli, S. Pennazzi, A. Sarti, and V. Vagnoni, CERN-LHCb-2007-059 (2007).

[6] V. Tisserand, Belle and BaBar Collaborations, Proceedings of 5th Flavor Physics and CP Violation Conference (FPCP 2007), Bled, Slovenia, 9 (2007).

[7] M. Gronau and D. London, Phys. Lett. B253, 483-488 (1991).

[8] M. Gronau and D. Wyler, Phys. Lett. B265, 172-176 (1991).

[9] D. Atwood, I. Dunietz, and A. Soni, Phys. Rev. Lett. 78, 3257-3260 (1997).

[10] A. Giri, Y. Grossman, A. Soffer, and J. Zupan, Phys. Rev. D68, 054018 (2003).

[11] M. Patel, CERN-LHCb-2006-066 (2006).

[12] M. Patel, CERN-LHCb-2007-043 (2007).

[13] J. Libby, A. Powell, J. Rademacker, and G. Wilkinson, CERN-LHCb-2007-098 (2007).

[14] V. Gibson, C. Lazzeroni, and J. Libby, CERN-LHCb-2007-048 (2007).

[15] S. Cohen, M. Merk, and E. Rodrigues, CERN-LHCb-2007-041 (2007).

[16] G. Wilkinson, CERN-LHCb-2005-036 (2005).

[17] V. Gligorov, CERN-LHCb-2007-044 (2007).

[18] R. Fleischer, Nucl. Phys. B671, 459-482 (2003).

[19] L. Fernandez, Acta Phys. Pol. B. 38, 931-940 (2006).

[20] D. Volyanskyy and J. Van Tilburg, CERN-LHCb-2007-027 (2007).

[21] S. Amato, J. McCarron, F. Muheim, B. Souza de Paula, and Y. Xie, CERN-LHCb-2007-047 (2007).

[22] Y. Xie, CERN-LHCb-2007-130 (2007).

[23] S. Amato, M. Gandelman, C. Göbel, and L. De Paula, CERN-LHCb-2007-045 (2007).

[24] O. Deschamps, S. Monteil, A. Robert, P. Perret, F. Machefert, P. Robbe, and M.H. Schune, CERN-LHCb2007-046 (2007). 